\title{
Once-weekly teriparatide administration for 24 weeks in postmenopausal women with osteoporosis
}

\author{
T. Kawada
}

Received: 8 March 2014 / Accepted: 15 April 2014 /Published online: 21 May 2014

(C) International Osteoporosis Foundation and National Osteoporosis Foundation 2014

\section{Dear Editor,}

Sugimoto and colleagues conducted a follow-up study of 28 postmenopausal women with osteoporosis to clarify the effect of once-weekly teriparatide administration for 24 weeks using several biologic markers [1]. After 24 weeks of treatment, significant increase of the serum osteocalcin, a bone formation marker, and significant decrease of the urinary deoxypiridinoline, a bone resorption marker, were observed. In contrast, no significant change of the serum procollagen type I N-terminal propeptide (PINP), a bone formation marker, or urinary cross-linked N-telopeptide of type I collagen (NTX), a bone resorption marker, was observed. I have two concerns on their study.

First, the authors conducted their study without setting a standard protocol for the daily teriparatide administration, which is described in the last paragraph of the Discussion section as a study limitation. They measured four biological markers at four time-points, including measurements before the start of treatment. Unfortunately, there are differences in the test situations in the past several studies, which makes the comparability not satisfactory. For example, the authors have quoted two references $[2,3]$ related to the effects of two treatment regimens on fracture risk reduction, and I have a question as to their reason for arriving at the conclusion of no difference in the relative risk reduction between 80 and $65 \%$ in these studies. There is no way to adjust for ethnic differences in the study, and comparison of data within the study should be conducted. In addition, the characteristics of their population should be checked for its representativeness of Japanese postmenopausal women.

A reply to this comment can be found at doi 10.1007/s00198-014-2722-5.

T. Kawada $(\bowtie)$

Department of Hygiene and Public Health, Nippon Medical School,

1-1-5 Sendagi, Bunkyo-Ku, Tokyo 113-8602, Japan

e-mail:kawada@nms.ac.jp
Second, distributions of PINP and NTX are logarithmicnormal in general, and Wilcoxon's matched-pair signed-rank test should be applied if Friedman's test with a strict criteria of alpha/3 shows significance. Alternatively, paired $t$ test with logarithmic transformation of variables should be conducted after repeated-measure analysis of variance. The number of patients in the study is limited $(n=28)$, and power analysis is indispensable for repeated measures [4].

Finally, the authors have mentioned in the last paragraph the advantage of sustainment for up to 72 weeks in their trial. But I feel that there is no clear evidence for their proposal. The lack of statistical significance does not mean the lack of clinical effectiveness, and setting of a control group as standard protocol should be considered for accurate analysis.

\section{References}

1. Sugimoto T, Nakamura T, Nakamura Y, Isogai Y, Shiraki M (2014) Profile of changes in bone turnover markers during once-weekly teriparatide administration for 24 weeks in postmenopausal women with osteoporosis. Osteoporos Int 25:1173-1180. doi:10.1007/ s00198-013-2516-1

2. Neer RM, Arnaud CD, Zanchetta JR, Prince R, Gaich GA, Reginster JY, Hodsman AB, Eriksen EF, Ish-Shalom S, Genant HK, Wang O, Mitlak BH (2001) Effect of parathyroid hormone (1-34) on fractures and bone mineral density in postmenopausal women with osteoporosis. N Engl J Med 344:1434-1441

3. Nakamura T, Sugimoto T, Nakano T, Kishimoto H, Ito M, Fukunaga M, Hagino H, Sone T, Yoshikawa H, Nishizawa Y, Fujita T, Shiraki M (2012) Randomized Teriparatide [human parathyroid hormone (PTH) 1-34] Once-Weekly Efficacy Research (TOWER) trial for examining the reduction in new vertebral fractures in subjects with primary osteoporosis and high fracture risk. J Clin Endocrinol Metab 97: 3097-3106

4. Jung SH, Ahn C (2003) Sample size estimation for GEE method for comparing slopes in repeated measurements data. Stat Med 22: $1305-1315$ 